\title{
¿Cómo abordar la investigación formativa desde los programas de ciencias básicas? Una propuesta con matemáticas y biografías.
}

\author{
Jeannette Vargas Hernández ${ }^{1}$
}

Liliana Caycedo Lozano ${ }^{2}$

\begin{abstract}
Resumen
Esta investigación de corte teórico, descriptivo y tipo cualitativo, abarca una revisión del género de las biografías y, al interior de las ciencias básicas, un estudio acerca del desarrollo de la investigación en enseñanza de las matemáticas. A partir de allí, se pretende establecer una propuesta marco, para los primeros semestres universitarios de pregrado, que genere un ambiente de investigación formativa, en el cual además de permitir a los estudiantes establecer un vínculo entre la historia de las ciencias y el aprendizaje de las mismas, los acerque a las diferentes fuentes de información y al uso de instrumentos; tanto para la recolección intencionada de dicha información como en la elaboración e interpretación de reportes de lectura.
\end{abstract}

Se considera la investigación formativa, como una función de la educación superior y planteamos centrar la atención en los procesos intencionados que se dan en el pre-grado, en los primeros semestres, para formar a los nuevos investigadores en el dominio de conocimientos, habilidades y destrezas que se exigen posteriormente, al estudiante, al formar parte de un semillero de investigación y al presentar una propuesta o proyecto de grado.

Palabras clave: didáctica de las matemáticas, procesos de enseñanza y aprendizaje, investigación formativa, biografías. 


\title{
$¿$ How to address educational research in the basic sciences subject matter? A proposal based on mathematics and biographies
}

\begin{abstract}
This theoretical, descriptive and qualitative research encompasses a review of the biography gender and, in the field of basic sciences, a study on the development of research in math teaching. Based on that, the aim is to deploy a frame proposal, for the first terms in undergraduate studies, resulting in an educational research environment, which besides allowing students to establish a link between the history of sciences and learning, will bring them closer to the varied sources of information and the use of tools; both for the targeted gathering of such an information and for preparing and interpreting reading reports.

Educational research is considered to be a feature of higher education, and we are proposing to focus in the targeted processes delivered throughout undergraduate studies, during the first semesters, to educate novel researchers in the mastering of some knowledge, skills and abilities, that will be further required, when the student will enter in a research breeding ground or will have to present his/her undergraduate proposal/project.
\end{abstract}

Palabras clave: didactics of mathematics, teaching/learning processes, educational research, biographies.

Recibido: 14 de octubre 2011 Aceptado: 01 de diciembre 2011

\section{lntroducción}

A partir de la década de los noventa, el impulso que en el ámbito universitario se ha pretendido dar a la cultura investigativa se ha hecho explícito, entre otros, en el espacio de la investigación formativa, generándose un reto acerca de cómo se pueden potenciar y gestionar, desde las facultades y programas, las ideas que surgen en los diferentes campos del conocimiento, para aportar en organizaciones locales que influyan en la propuesta curricular institucional. 
Es de esperar que frente a este reto la comunidad académica, en especial los docentes y los docentes investigadores, aúnen esfuerzos para generar propuestas sistemáticas e interdisciplinarias en donde aporten sus conocimientos y experiencias desde sus perfiles específicos. Frente a ello nuestra propuesta de las biografías, como instrumento en investigación formativa, consiste en plantear una de esas organizaciones locales que nace en el programa de ciencias básicas y puede ser ajustada en los diferentes programas académicos.

De esta manera planteamos una investigación que tiene como objetivo el formular una propuesta de investigación formativa con las biografías como instrumento didáctico, la cual se centra en la intención de generar habilidades de búsqueda de información y comunicativas ligadas a la investigación, para facilitar la indagación en cualquier campo científico.

Esta investigación acoge la postura de un profesor como profesional reflexivo, quien en sus procesos de transición a las nuevas exigencias de su gestión en el aula, de manera específica, de su papel como formador de investigadores, está en la posibilidad de generar y apropiarse de conocimientos tanto en las fases de planeación como en su gestión en el aula.

\section{Referentes teóricos}

La Universidad no se limita a los estamentos que la configuran: docentes, estudiantes, administrativos y lugar donde se agrupan, sino que éstos se interrelacionan de tal manera que la producción de saberes, la expresión y confrontación de los mismos, en un clima de libertad y de búsqueda otorgan el sentido a la Universidad. Los elementos que hacen que la universidad tenga esta categoría son: la generación, expresión y difusión libre del conocimiento en interacción, en todas las áreas del mismo y en las artes, sin excepción. La Universidad es un ente de cultura porque en ella se apoya, ahí se forja y ella misma la transmite.

En los inicios de las Universidades Europeas, en esa Europa medieval... las Universidades surgen como continuación de las escuelas que unidas a las iglesias, conventos y catedrales se encargaban de la producción y difusión del conocimiento. Estas escuelas se convirtieron en «estudios generales» por la diversidad de nacionalidades de los alumnos y maestros pero sobre todo por la variedad de conocimientos que ahí se impartían; "es un lugar en el que se agrupan miles de escolares, en el que el entendimiento puede confiadamente desplegarse y especular, seguro que encontrará adversarios y jueces en el tribunal de la verdad. Es un lugar donde la investigación es estimulada y los descubrimientos adquiridos y perfeccionados vuelven inofensiva la temeridad; el error se manifiesta mediante el choque de entendimiento contra entendimiento, de conocimiento contra conocimiento» (Mayagoitia, 1945: 32). 
Parafraseando a Corral, (1999), un requerimiento que se considera debe ser atendido en una universidad que se desenvuelve en y para la cultura, es la investigación. Gracias a este ejercicio permanente de todos los integrantes de esta institución, se logra trascender los límites del conocimiento. Este es el verdadero objetivo que persigue la investigación y con ella se transforma la vida de la universidad. No habrá cultura, ni habrá posibilidad de difundirla, si no se encuentra presente esta búsqueda permanente.

\section{Investigación formativa}

Como una manifestación de la cultura investigativa, a partir de la segunda mitad de la década del 90, en las instituciones de educación superior, de nuestro país, se comenzó a hablar de investigación formativa como aquel tipo de investigación que se hace entre estudiantes y docentes en el proceso de desarrollo del currículo de un programa, y que es propio de la dinámica de la relación con el conocimiento que debe existir en todos los procesos académicos tanto en el aprendizaje, por parte de los estudiantes, como en la renovación de la práctica pedagógica por parte de los docentes (Restrepo, 2007).

Esta función de la educación superior, la investigación formativa, tiene varias acepciones, dentro de las cuales, proponemos centrar la atención en la investigación formativa como los procesos intencionados que se dan en el pre-grado para formar a los nuevos investigadores en el dominio de conocimientos, habilidades y destrezas que les permitan desarrollar la investigación con todo el rigor.

Nuestra propuesta se organiza e identifica con el concepto del Consejo Nacional de Acreditación (CNA, 1998). Por ello, se asume la investigación formativa como el espacio que busca formar en y para la investigación a través de actividades que no hacen parte necesariamente de un proyecto concreto de investigación. Su intención es familiarizar al estudiante con la investigación, su naturaleza, sus fases y funcionamiento.

De acuerdo con Redcolsi -Red Colombiana de semilleros de investigación- se entiende que la investigación formativa es una de las maneras de desarrollar la cultura investigativa, el pensamiento crítico y autónomo, que permite a estudiantes y profesores acceder a los desarrollos del conocimiento y a la realidad internacional, nacional y regional.

También se considera a la investigación formativa como un conjunto de prácticas, que le permiten al estudiante generar y desarrollar su competencia investigativa para y en la investigación, ya sea:

A través de su vinculación a un grupo de investigación; realizando en éste tareas propias de la investigación como puede ser la búsqueda de información 
bibliográfica, la participación en discusiones del equipo de investigación (seminarios, reuniones de balance, de seguimiento, etc.), o en el desarrollo de su propio proyecto de grado que debe tener algún fundamento u orientación investigativa y al participar en los club de lectura, club de traducciones, grupos de estudios o cuando es integrante de semilleros de investigación.

Se encuentra también posturas que afirman que la lógica de los procesos de investigación, así como las herramientas conceptuales, metodológicas y técnicas para desarrollarla, pueden enseñarse. Dicho con otras palabras: se puede formar para la investigación (Ossa, 2002; Parra, 2004).

\section{Biografía}

Las biografías constituyen un género histórico-literario específico en el que un investigador «reconstruye» una trayectoria individual sobre la base de documentación preferentemente escrita y con el auxilio eventual de fuentes orales, en caso que se trate de la biografía de una persona contemporánea.

Normalmente el género biográfico se orienta hacia personajes históricos relevantes y públicos por su aportación en los campos de la política, la ciencia o el arte, entre otros. A lo largo de este siglo, se ha consolidado una galería de mitos culturales surgidos esencialmente del campo del deporte y del arte (música y cine) que han servido de base para la publicación de biografías, a menudo polémicas, al servicio de la consolidación mediática de esos mismos mitos.

La Biografía como una de las formas más antiguas de expresión literaria, es la revisión escrita de la vida de una persona. Carmen Bravo-Villasante (Madrid, 1918-1994), filóloga, folclorista y traductora española, pionera en el estudio universitario de la literatura infantil, quien cultivó extensamente el género de la biografía, interesándose de manera especial en personajes literarios del siglo XIX, la identifica en literatura como el retrato en pintura. De esta manera afirma que lo mismo da ver el retrato del Duque de Goya, que leer la biografía del mismo Duque, hecha por un buen biógrafo. «La biografía, en toda su complejidad, representa a todos los géneros literarios. La biografía es historia, poesía, novela, cuento, ensayo, crítica, erudición y filosofía, un vasto mundo complejo, abarcador, en el que se entrelazan todas las manifestaciones literarias que acostumbramos separar en géneros» (Bravo, 1969: 13).

Continúa esta filóloga afirmando que sabemos que el retrato en pintura es un arte, el cual exige la veracidad del parecido y luego la libertad de la interpretación, y así, sucede en la biografía en donde se exige la autenticidad de los hechos y luego viene la interpretación artística. Hechos e interpretación en donde vida y obra van unidas indisolublemente; no hay vida externa sin vida interna. Bravo (1969) ejemplifica, esta unión indisoluble, afirmando que 
el relato biográfico de un escritor tendrá siempre que referirse a su obra. Un escritor va por la calle pensando en sus libros; entra en el sueño pensando en su novela; en su poesía, si es poeta; se levanta y escribe su diario. Todos o casi todos sus movimientos están encaminados hacia sus libros, y su vida entera está teñida de literatura, como se supone que un pintor verá cuadros en todos los atardeceres.

Adiciona a lo anterior que el biógrafo para escribir una biografía necesita persistencia, una constancia imponente y tener la fuerza de una hormiga que amontona brizna tras brizna en su hormiguero, que son los papeles de los archivos y los epistolarios, entre otros; pues el biógrafo en efecto, es historiador y erudito (Bravo, 1969:15).

Al emprender una mirada sobre la Biografía y la Historia, se está en el terreno de lo que pueden llamarse tipos historiográficos y se considera imperativo para ello consultar una de las obras de José Luis Romero (1909-1977), uno de los más grandes historiadores latinoamericanos.

Romero al referirse a la biografía la ubica como un tipo historiográfico (1945), definiendo éste como cierto esquema regular dentro del cual se ordenan y estructuran los elementos de la intelección histórica, valorados de acuerdo con los elementos históricos que se eligen para formular la conceptualización. De tal manera que sitúa a la biografía en el tercer tipo historiográfico, que se caracteriza por partir de la intuición del individuo como sujeto de un devenir histórico.

En la vida del individuo, el plazo del devenir histórico aparece precisado por el nacimiento y la muerte, y proporciona un esquema suficientemente simple para la ordenación del proceso histórico en una sucesión de etapas que se manifiestan como decisiones y acciones individuales.

Con otra mirada si se va a considerar una especie de «taxonomía» de las biografías, teniendo presente que la forma de etiquetarlas no se desarrolla en términos categóricos, sino más bien, son nombres que sugieren algún tipo de énfasis o punto de vista privilegiado por el biógrafo.

Esta tarea se toma de la presentación que hace Edgar de Jesús Velásquez, investigador en los ámbitos de la biografía y la historia política de Colombia y América Latina, Siglo XX. Velásquez (2007) que señala alcances limitaciones y problemas de cada uno de los tipos biográficos, describe así la biografía histórica, la psicológica, tendenciosa, imparcial, auténtica, novelada, científica, mediática, confesional, sectorial, integral, comparada, oral, crítica y la biografía desde abajo.

Por los intereses de este trabajo nos detenemos, literalmente en Velásquez, así:

La biografía histórica. Consiste en una relación cronológica de los hechos y debe tener dos requisitos: la cronología lineal precisa y la autenticidad 
de los testimonios y documentos. La biografía histórica es analítica y descriptiva. Para este tipo de biografía es esencial la precisión cronológica en el orden de sucesión de los hechos y eso le imprime un rasgo esencial, como el carácter lineal de la existencia del biografiado. Desde ese punto de vista, la biografía histórica es la paciente acumulación de los hechos en su forma de ocurrencia (Velásquez, 2007: 78).

La biografía psicológica. Revela rasgos caracterológicos del sujeto y tiene como característica esencial acumular los datos suficientes para conocer la personalidad del biografiado. En el marco de la biografía psicológica se pueden privilegiar facetas como la vida pública, privada o sentimental del biografiado, haciendo énfasis en la reacciones del mismo frente a las situaciones dadas. Los datos o hechos del biografiado, se constituyen en insumos para quien opta por la biografía psicológica, pues a partir de ellos, intenta aproximarse a un conocimiento amplio de la vida del biografiado. Así por ejemplo, para quien decida llevar a cabo una biografía de corte psicológico, obviamente, además de tener competencias en el campo de la psicología, será de vital importancia disponer de suficiente información de cada una de las etapas del ciclo vital del sujeto, y lograr inferir a partir de ellas, las incidencias con el contexto. Por la vía de la biografía psicológica es posible comprender las patologías y los comportamientos atávicos de gobernantes, por cuyas acciones polarizan a la sociedad entre quienes los admiran y los odian (Velásquez, 2007: 79).

La biografía cientifica. Este tipo de trabajo considera al biografiado en el estado de la sociedad correspondiente y para tal efecto, tiene en cuenta los factores hereditarios y ambientales. Las investigaciones adelantadas por los científicos sobre el genoma humano, las enzimas y también los descubrimientos de las múltiples incidencias del ambiente sobre los individuos de una población y poblaciones en general, son importantes fuentes para quien decida realizar una biografía científica sobre un sujeto. $\mathrm{La}$ biografía científica además de ser sintética, es esencialmente educativa, porque con su método genético nos da a conocer el determinismo de la conducta humana; las causas de los errores, de los fracasos y de los triunfos. $\mathrm{La}$ biografía científica debe explicar el porqué de las acciones, sentimientos, de los hábitos y de los comportamientos. Desde la perspectiva de este tipo de biografía, es posible demoler mitos y creencias sobre las razas y los determinismos habiendo servido de base para la opresión, la eliminación o la exclusión de culturas y pueblos enteros.

El biógrafo interesado en la biografía científica deberá inicialmente medir sus competencias y facultades, en virtud al lenguaje sobre el cual está situada la información y por ello mismo, dada la forma y el contenido de narración; es un lenguaje especializado del campo de la medicina, la biología, la química y la genética (Velásquez, 2007: 87). 
El término biografía, etimológicamente proviene del griego «bíos» vida, más el sufijo «grafía» de la raíz «graf», cuyo significado es «tratado». Por ello, la biografía puede ser definida como el tratado o la historia de vida de una persona, y algunos de los sinónimos atribuidos son semblanza, perfil y trayectoria.

\section{Metodología de la investigación}

El documento que ahora se elabora forma parte de una investigación más amplia de corte teórico, descriptivo y tipo cualitativo. En una serie de fases en esta investigación se realizó la presentación de la problemática; estado de la cuestión, al igual que la elaboración de un marco referencial que incluyó: Investigación. Cultura investigativa. Investigación formativa. Género biográfico. Trayectorias del género biográfico. Clasificación de este género. Biografías. Autobiografías. Historias de Vida. ${ }^{3}$

También a partir de los referentes teóricos se identificó la habilidad; lectura interpretativa y forma de abordarla y a su vez se realizó una revisión y elaboración de los antecedentes de la Investigación Formativa en Colombia, América y en la Institución. Las fases finalizaron con el planteamiento de la propuesta y la presentación de un ejemplo práctico con biografías en el componente temático de matemáticas y de química. ${ }^{4}$

\section{Propuesta teórica desde matemáticas}

\section{Marco referencial específico}

Esta investigación de corte teórico tiene como uno de sus objetivos realizar una propuesta sobre enseñanza y aprendizaje, la cual se enmarca en la investigación formativa e incluye elementos de la Didáctica de la matemática. Con la intención de situar la perspectiva del trabajo, se presenta el siguiente examen sobre la investigación en enseñanza:

La literatura de investigación en educación muestra que hace no tanto tiempo la enseñanza se consideraba un arte y, en consecuencia, era difícil de analizar, intervenir y someter a ciertas reglas. La manera de concebir al profesor y la enseñanza, desde la teoría y la investigación educativa, ha sufrido una evolución que se puede ver en términos del objeto específico de estudio o de la complejidad considerada. 
Por ejemplo, Rosenshine (1979) señala que inicialmente se asoció la buena enseñanza con características personales del profesor, luego el interés se centró en la interacción entre maestro y estudiantes, y posteriormente los estudios enfocaron al estudiante $\mathrm{y}$, en particular, al dominio de un cierto contenido por parte de los estudiantes. Medley (1979) muestra que la investigación enfocada en la eficacia del profesor ha considerado características personales y profesionales, métodos usados para enseñar, conductas y clima del aula, y competencias del profesor.

Por su parte, Koelher y Grouws (1992) organizando los estudios sobre la enseñanza de las matemáticas y sus efectos, identifica cuatro niveles de complejidad. En el primero se considera la efectividad del maestro relacionada con rasgos personales como buen juicio, consideración, entusiasmo, magnetismo personal, apariencia personal y lealtad. En el segundo se incluyen estudios que involucran observaciones múltiples del aula que proporcionan detalles de la instrucción en matemáticas. En el tercero se amplía la mirada a los resultados del estudiante y se incluyen tanto actitudes como logros; se considera que características del estudiante (e.g., género, raza, nivel de confianza) pueden afectar la práctica del maestro y las acciones del estudiante mismo. El cuarto nivel recoge estudios en los que se reconoce cabalmente la complejidad de la enseñanza de las matemáticas en términos de una diversidad de aspectos que actúan interconectadamente (e.g., rango amplio de niveles de habilidad a lograr, rango amplio de temas para enseñar, surtido muy amplio de metodologías entre las cuales escoger); adicionalmente hay técnicas de recolección y análisis de datos más sofisticadas que permiten abordar preguntas de investigación más complejas.

Probablemente, el cambio más sustancial logrado en los estudios de este nivel radica en la consciencia que se tiene de la necesidad de hermanar la investigación en enseñar con la investigación en aprender.

En el marco que se acaba de esbozar, en la década de 1980, y a partir de autores como Donald Schön, se asume que los profesores generan conocimiento sobre la enseñanza a partir del trabajo práctico que realizan al enseñar en aulas particulares, y que ese conocimiento amerita ser investigado. Al mismo tiempo surge con fuerza el interés en las creencias, desde diversas disciplinas (e.g., psicología, ciencias políticas, antropología, educación). Entre los educadores, el interés en el estudio de las creencias y concepciones de los profesores lo alimentó el cambio de paradigma, el pasar de concebir al profesor como un técnico a concebirlo como un ser pensante, reflexivo.

Así, a finales de la década de 1990 surge el interés por comprender la gestión del docente en el aula y comienza a considerarse la importancia de analizar la actividad de los docentes en el aula, de igual manera las creencias y las concepciones adquieren un papel prominente como base para estudiar a los profesores de matemáticas y su enseñanza. 
Según Sánchez (2010, 2011), en la actualidad, en el campo de la educación matemática se percibe un aumento de los estudios que investigan las creencias del profesor lo mismo que aspectos particulares de las prácticas de los docentes en el aula (e.g., decisiones fuera del aula de clase, acerca de los recursos usados por los profesores para definir el contenido de las lecciones, sobre aspectos concretos de las prácticas de los docentes en el aula; por ejemplo los tipos de preguntas formuladas durante las clases). Sin embargo, es necesario ampliar la investigación en el campo a nuevas temáticas (Camacho, 2011) y de manera que los problemas de investigación se sitúen en el nivel universitario (Moreno, 2011).

En la panorámica sobre investigación en enseñanza, que se presenta en los párrafos anteriores, subyace la idea y evolución del término didáctica, que desde el punto de vista clásico supone el saber didáctico como un saber técnico, teniendo por objetivo inmediato proporcionar al profesor los recursos técnicos que éste necesita para llevar a cabo su labor de la manera más satisfactoria posible, hasta alcanzar actualmente la idea de la didáctica de las matemáticas que incluye el conocimiento matemático entre sus objetos de estudio.

Esta investigación acoge que el profesor como un profesional reflexivo, en sus procesos de transición a las nuevas exigencias de su gestión en el aula, de manera específica de su papel como formador de investigadores, está en la posibilidad de generar y apropiarse de conocimientos en las fases de planeación y gestión en el aula.

La didáctica de las matemáticas es la ciencia del estudio y de la ayuda al estudio de las matemáticas. Su objetivo es llegar a describir y caracterizar los procesos de estudio - o procesos didácticos - de cara a proponer explicaciones y respuestas sólidas a las dificultades conque se encuentran todos aquellos (estudiantes, profesores, padres, profesionales, etc.) que se ven llevados a estudiar matemáticas o a ayudar a otros a estudiar matemáticas (Chevallard et-al., 2000: 60).

Con la claridad del amplio mundo de fenómenos que implica en la actualidad la disciplina Didáctica de la matemática y la conciencia de no entrar en profundidad, se tomará de su campo algunas posturas que se consideran necesarias y compatibles en el discurso que se está presentando.

Al retomar una definición de didáctica de la matemática, es importante observar que se redefine y se rescata el estudio $^{5}$ de las matemáticas como el eslabón perdido entre la enseñanza y el aprendizaje. Considerando el estudio no sólo como esa actividad que uno realiza en solitario fuera de clase, sino

$5 \mathrm{El}$ estudio es hoy el eslabón perdido entre una enseñanza que parece querer controlar todo el proceso didáctico y un aprendizaje cada vez más debilitado por la exigencia de que se produzca como consecuencia inmediata, casi instantánea, de la enseñanza (Chevallard et.al., 2000:. 13). 
en todo espacio y momento, de manera general cuando decimos «él estudia ingeniería». Por lo tanto en este contexto, la enseñanza aparece como un medio para el estudio.

En la propuesta teórica acerca de la investigación formativa en los primeros semestres de pregrado; matemáticas desde el programa de ciencias básicas, se tienen en cuenta las creencias sobre la naturaleza de las matemáticas y dos delineamientos que se presentan desde la didáctica de la matemática; en este último aspecto se plantea que la organización de la enseñanza al tener en cuenta lo que los estudiantes tienen en común, puede potenciar la formación de grupos de estudiantes capaces de estudiar juntos puesto que «la organización de la enseñanza debe basarse más en lo que los estudiantes tienen en común que en lo que es particular a cada uno de ellos. Desde un punto de vista antropológico, el estudio y, con él, el aprendizaje son actividades que unen a los individuos» (Chevallard et.al2000: 200).

Aunado a lo anterior, la tarea de los centros de enseñanza y, en particular, de los profesores es la de crear las mejores condiciones posibles para que los estudiantes puedan estudiar - y por lo tanto aprender -. Para ello los centros docentes disponen de un conjunto de dispositivos (bibliotecas, aulas, tutorías, actividades extraescolares, salas de Internet, entre otros).

En nuestra investigación asumimos que la enseñanza de las matemáticas tiene, hoy en día, una importancia real en la educación de las nuevas generaciones, debido a que las matemáticas son una de las expresiones culturales básicas de nuestra actual sociedad. Las matemáticas expresan necesidades culturales básicas, pero no tienen igual intensidad ni idéntico desarrollo en comunidades diferentes. Las matemáticas son uno de los elementos esenciales de la cultura de nuestra época, y son mucho más que una disciplina formal, constituyen una de las formas básicas del conocimiento que permiten comunicar, interpretar, predecir y conjeturar (Rico, 1989: 37).

Además de lo expresado en el párrafo anterior es necesario tener presente que hay investigadores que sugieren que la mayoría de la gente percibe a las matemáticas como un conjunto fijo de conocimientos pulidos y acabados. Es una disciplina fría y austera que le da poco espacio al juicio y a la creatividad (Barbeau, 1989). Otros por su parte han reseñado estudios que documentan cómo los docentes difieren ampliamente en sus creencias sobre la naturaleza y el sentido de la matemática. Las diferencias observadas van desde considerar la matemática como un cuerpo estático y unificado de conocimientos absolutos e infalibles, hasta considerarla un campo de la creación y la invención humana en continua expansión (Thompson, 1992).

Frente a este aspecto en nuestra propuesta se busca tener implícita la mirada a las matemáticas como una disciplina falible, cambiante y similar a otras 
disciplinas por ser un producto de la inventiva humana. Este punto de vista dinámico acerca de las matemáticas como un producto que no está terminado, sino que es una disciplina que está avanzando constantemente y reajustándose a nuevas situaciones (Ernest, 1989), se puede promover entre los estudiantes, a través del acercamiento a las biografías y, por ende, a obras de personajes vinculados con el desarrollo de conceptos e ideas matemáticas.

\section{La propuesta}

Se parte de considerar que existen múltiples opciones para organizar el trabajo de las biografías, encaminado a la mirada en investigación formativa. En esta propuesta se pueden establecer muchas facetas consideradas óptimas para el ejercicio con los estudiantes de primeros semestres de programas de pregrado.

A partir de un documento inicial seleccionado por el docente, los estudiantes elijen un personaje, que sea de su interés, para consultar la biografía. Realizan lecturas que les permiten organizar una semblanza del personaje y entre los documentos consultados se incluye la lectura de un documento escrito por el propio personaje.

El docente en su respectivo momento hace entrega del documento inicial, ${ }^{6}$ la ficha topográfica (figura 6) y la ficha de trabajo (figura 7).

\begin{tabular}{|c|c|}
\hline No. ITEM & INFORMACIÓN \\
\hline 1. Título completo: & \\
\hline 2. Tipo de documento: & \\
\hline 3. Autor: & \\
\hline 4. Perspectiva Disciplinar: & \\
\hline 5.Lugar y fecha: & \\
\hline 6.Editor: & \\
\hline 7.Volumen: & \\
\hline 8. Páginas: & \\
\hline 9. Descriptores o palabras claves: & \\
\hline 10.Ubicación: & \\
\hline
\end{tabular}

Figura 6.

Ficha de trabajo.

\begin{tabular}{|l|l|}
\hline Descripción del documento & Interpretación del analista \\
\hline & \\
\hline
\end{tabular}

Figura 7.

6 El las experiencias de aula se utilizó el artículo Forjadores del desarrollo de las matemáticas en Colombia: Otto de Greiff Haeusler. 
Los momentos por los que el estudiante navega en esta propuesta son:

1. Visitas a bibliotecas, para conocer sus servicios. Entrega de informe.

2. Lectura del documento inicial y socialización al interior de todo el grupo.

3. Organización de subgrupos de acuerdo con el personaje seleccionado.

4. Primera socialización de informes sobre el personaje seleccionado.

5. Selección de una obra de ese personaje.

6. Entrega de ficha topográfica como elemento que organiza la búsqueda bibliográfica.

7. Segunda socialización haciendo uso de la ficha topográfica.

8. Entrega, por parte del docente, de ficha de trabajo como elemento para la lectura interpretativa. Selección de monitores.

9. Tercera socialización de los avances utilizando para ello las fichas de trabajo.

10. Socialización y primera entrega de biografía.

11. Socialización de la lectura sobre obra escrita por el personaje.

12. Entrega final.

Las entregas parciales y socializaciones tienen en cuenta que la entrega final que contiene la semblanza del personaje seleccionado debe incluir:

Datos relacionados con fechas y eventos más significativos en la vida del personaje.

Estudios cursados.

Actitudes y habilidades del personaje.

Otros rasgos característicos de su personalidad.

Un título que represente o identifique muy claramente al personaje.

Interpretación o mensaje que la vida de este personaje le envía a la sociedad o al lector (estudiante) en particular. 
Resumen de elementos de la vida del personaje ya sea a través de un crucigrama, una historieta, o la forma artística que el estudiante elija.

Ejemplo práctico: un desarrollo de la propuesta desde matemáticas

En el desarrollo de la propuesta, se describen los pasos y algunos de los momentos; a través de trabajos elaborados por los estudiantes en dos semestres diferentes, II2004, I-2005. Sin embargo, es importante anotar que muchas de las estrategias de búsqueda bibliográfica y lectura interpretativa surgen desde 2003 (Vargas, y Caycedo, 2006) y son reelaboraciones tanto de prácticas como de observaciones en diferentes semestres y universidades (Vargas, y Garcharná, 2008).

Desarrollo y algunos productos.

Los momentos por los que se navega en esta propuesta:

Paso 1. La propuesta de acercamiento a la búsqueda bibliográfica inicia con la invitación a los estudiantes de primer semestre, para que conformen grupos de cinco o seis integrantes y elijan una biblioteca de una niversidad pública o privada, o una biblioteca de la Red de Bogotá. Luego de seleccionada la biblioteca, los estudiantes deben proceder a informarse sobre los requisitos para poder ingresar físicamente, no virtualmente. El día de la visita, centran su atención en los servicios que presta la biblioteca y recogen información en torno de las revistas de educación matemática y los volúmenes que allí se encuentren.

El primer reporte escrito debe contener información sobre los servicios que son ofrecidos en la biblioteca y las formas de acceder a ellos, e incluir una tabla en donde presenten nombre de las revistas y datos generales concernientes a ellas. La puesta en común de esta experiencia, se lleva a término en el horario de tutoría grupal.

Pasos 2 y 3 . El desarrollo de la propuesta continúa con la lectura del documento Forjadores del desarrollo de las matemáticas en Colombia: Otto de Greiff Haeusler (1995), escrito por Clara Helena Sánchez, doctora de la Universidad Nacional de Colombia. En este texto el esquema de la narración en donde intervienen y confluyen diferentes personajes de la vida política y académica de Colombia permite una amplia y flexible selección de «vidas» y temáticas.

Los estudiantes realizan la lectura de este documento inicial y lo socialización con todo el grupo. Aprovechando esta socialización para la organización de subgrupos de acuerdo con el personaje seleccionado, quien puede ser una de las figuras, ya sea político, o científico, entre otros, mencionado en el artículo y que por algún motivo, la indagación sobre su vida, resulte de interés para el grupo de estudiantes. 
A la vez que se obtendrán datos cronológicos y sobre sus rasgos de personalidad, la principal indicación para esta búsqueda sobre la vida y obra del personaje elegido consiste en detenerse a examinar una obra escrita por él, que sea comprensible e interesante para el grupo.

Pasos 4, 5 y 6 . Los estudiantes realizan la primera presentación verbal sobre la vida del personaje seleccionado, permitiendo para ello que utilicen los apuntes y herramientas que consideren necesarias. De acuerdo con esta primera mirada al personaje, el profesor sugiere se seleccione una obra escrita por aquél. El docente hace entrega de ficha topográfica como elemento que organiza la búsqueda bibliográfica.

Paso 7. A partir de la ficha topográfica entregada por el profesor, se puede observar lo siguiente: Un grupo que escogió como personaje al profesor Yu Takeuchi y está consultando su obra «¿Por qué la Matemática es importante?», además de incluir en su presentación, de avances, algunos de los rasgos solicitados, diligenció la ficha topográfica así:

Al finalizar la socialización, el profesor indaga sobre los beneficios que los estudiantes recibieron al usar la ficha topográfica, respecto a la forma como ellos venían trabajando y aprovecha para hacer entrega de la ficha de trabajo para organizar la lectura interpretativa que se está llevando a término.

Paso 8. Este momento es relevante, puesto que aquí se selecciona un monitor por cada 4 grupos que se encarga de recibir en su correo electrónico tanto las fichas topográficas como las fichas de trabajo y el avance de la semblanza del personaje. La principal tarea es observar que todos los grupos hayan diligenciado al menos dos fichas topográficas entre las cuales se debe encontrar una relacionada con la obra del personaje y las demás referidas a su biografía, cada una de ellas acompañadas de su correspondiente ficha de trabajo. Cada grupo debe anexar la semblanza del personaje, de la manera como esté elaborada hasta ese momento. Todo ello será enviado al docente con copia al monitor.

Así tendremos en el caso la ficha de trabajo y en la figura 11 un borrador de la biografía, de la consulta sobre el matemático Julio Garavito Armero:

Paso 9. Cuando los monitores han verificado la entrega de fichas y avance en la semblanza del personaje, se puede realizar la tercera socialización, y utilizar para ello las fichas de trabajo, que han sido previamente revisadas por el profesor, exigiendo elementos de redacción y profundidad en sus análisis y expresión del mensaje que este grupo desea resaltar sobre la vida de este personaje.

Paso 10. Para esta socialización y primera entrega de la biografía, se organiza en clase la elaboración de carteleras bajo la mirada cronológica de acontecimientos en la vida de cada personaje. 
Se puede sugerir que las carteleras tengan un título que identifique al personaje, ya sea por su personalidad, su forma de vida o de estudio. Los grupos rotan por los carteles expuestos, encontrando semejanzas y diferencias entre los diferentes personajes. La identificación de cada grupo con su personaje investigado, es notoria, no solamente porque buscan la manera de hacer sobresalir sus obras sino también sus anécdotas y características personales, siendo fácil escuchar entre ellos las expresiones: ¿cómo pudo aprender tres idiomas y también saber de música y matemáticas? En este estadio ya hay mezclas de actitudes de admiración, comparación y propósitos, entre otros.

Paso 11. Socialización de la lectura sobre obra escrita por el personaje. Se realiza la socialización tanto con la ficha topográfica como con la ficha de trabajo y se hace rotando grupos de dos en dos hasta que la rotación permite que todos los grupos tengan una idea de la obra estudiada por los demás equipos de trabajo.

Paso 12. En la fecha de entrega final, todos los documentos deben ser enviados por correo electrónico al profesor y a los monitores, junto con la semblanza final que puede ser a manera de un trabajo creativo sobre su personaje, ya sean caricaturas, crucigramas, cuentos, entre otros.

\section{Notas finales}

Esta investigación tiene una gran riqueza de matices con los que se espera que estudiantes y profesores tengan la oportunidad de iniciar un mayor acercamiento a la investigación formativa a través de una mezcla de literatura, historia, ciencias exactas y experimentales.

Se asume a la biografía y su orientación hacia personajes históricos y públicos, relevantes por su aportación en los campos de la política, la ciencia o el arte, entre otros, como una herramienta didáctica que rescata y potencia la valoración de unos talentos que se pueden colocar como «puntos de referencia», «elementos inspiradores» o «experiencias motivadoras» en los proyectos de vida (tanto personales como profesionales) de los estudiantes en esta etapa de su formación, caracterizada en la mayoría de veces por la inexistencia de unos «patrones paradigmáticos de peso» que permitan orientar adecuadamente sus proyectos, dada la proliferación de un sinnúmero de propuestas de estilos de vida carentes de fundamento y de valores trascendentales.

La puesta en marcha de esta propuesta de investigación formativa no se restringe a un solo semestre, ni a una sola facultad, por el contrario, se considera que de manera simultánea en otros componentes temáticos, o con los productos obtenidos en el primer semestre, pueden los docentes y estudiantes continuar la profundización y aprendizaje en los semestres y componentes temáticos siguientes. 
Mas allá de los escritos que presentan los estudiantes, como resultado de sus consultas, se encuentra toda la riqueza de expresiones y vivencias en los momentos de las puestas en común o socializaciones de las lecturas que se han llevado a término, por ello se considera que estos son los momentos a privilegiar para los aspectos en los cuales el profesor desee poner acento, ya sean de carácter axiológico, científico o técnico, entre otros.

\section{Referencias bibliográficas}

Artzt, A. 1999. "A Structure to Enable Preservice Teachers of Mathematics to Reflect on Their Teaching”. Journal of Mathematics Teacher Education, 2(2): 143-166.

Barbeau, E.J. 1989. Matemáticas para el público. Documento presentado en la reunión de la Comisión Internacional de Instrucción Matemática. Leeds, Inglaterra: Universidad de Leeds.

Bravo-Villasante, C. 1969. Biografía y Literatura. Barcelona: Plaza \& Janes.

Camacho, M. 2011. «Investigación en didáctica de las matemáticas en el bachillerato y primeros cursos de universidad». En Investigación en Educación Matemática XV. Simposio de la SEIEM. Marín, M. G. Fernández, L. Blanco y M. Palarea (Eds.). 195- 226. Ciudad Real: Universidad de Castilla.

CNA. 1998. La evaluación externa en el contexto de la acreditación en Colombia. Santafé de Bogotá: Corcas.

Corral, G. 1999. ¿Qué es Universidad? México: Universidad Autónoma del Estado de México.

Chevallard, Y., M. Bosh, y J. Gascón. 2000. Estudiar Matemáticas. El eslabón perdido entre la enseñanza y el aprendizaje. Barcelona: Editorial Horsori, Universidad de Barcelona.

Ernest, P. 1989. "The Knowledge, Beliefs, and Attitudes of the Mathematics Teacher: a model". Journal Education for Teaching. 15(1): 13-33.

Fennema, E. y M. Loef. 1992. "Teachers' Knowledge and its Impact”. En D.A. Grouws (ed.). 147-164. Handbook of Research of Mathematics Teaching and Learning. New York: Mac Millan.

Koelher, S. M. y D. A. Grouws, 1992. "Mathematics Teaching Practices and their Effects". En D. Grouws (ed.) 115-126. Handbook of Research of Mathematics Teaching and Learning. New York: Mac Millan. 